\title{
An Optimal Control Problem in Closed-Loop Neuroprostheses
}

\author{
Gautam Kumar, Vikram Aggarwal, Nitish V. Thakor, Marc H. Schieber and Mayuresh V. Kothare ${ }^{1}$
}

\begin{abstract}
A control-theoretic study of complex systems such as closed-loop neural prostheses exhibits several challenges, from the design of an optimal feedback control problem to its solution. In this paper we study one such system, a closed-loop voluntary movement of a prosthetic finger using electrophysiological activities of a single cortical motor neuron. We develop an optimal feedback control problem in the nonlinear receding horizon based terminal set constraint framework. We analyze the feasibility and stability of the control problem. Further, we solve the control problem numerically by implementing a local optimum based nonconvex nonlinear programming algorithm. Finally, we study effects of visual and proprioceptive feedback pathways on the closed-loop system. Our results elucidate the importance of multiple feedback paths in designing a closedloop neural prosthetic system.
\end{abstract}

\section{INTRODUCTION}

Motor intended neural prostheses, such as artificial limbs, are driven and controlled by neural activities from the primary motor cortex (M1). An interface that connects these devices to the brain is called brain-machine interface (BMI). This interface contains a noise filter, a decoder to extract motor intended information from the activities of M1 neurons, a local prosthetic device movement controller and finally an encoder to feedback mechanical information, available from the prosthetic device, to the brain [1]. Thus the brain, the BMI and the prosthetic device together act as a closed-loop neural prosthetic system. In this paper, we study this system in an optimal feedback control framework. Our goal is not to model the exact system or demonstrate how the brain controls voluntary movements but to study the importance of proprioceptive as well as visual feedback in designing a closed-loop neural prosthetic system.

Motor intended neural prosthetic systems have been studied extensively in the last two decades. Most of these studies are either human clinical trials or animal trials [2]. Moreover, these studies neglect the effect of proprioceptive feedback in designing the prosthetic system. It is well known

Gautam Kumar is with the Chemical Process Modeling and Control Center, Department of Chemical Engineering, Lehigh University, Bethlehem, PA 18015, U.S.A., gautam.kumar@lehigh. edu

Vikram Aggarwal is with the Department of Biomedical Engineering, Johns Hopkins University, Baltimore, MD 21205, U.S.A., vaggarwalejhu.edu

Nitish V. Thakor is with the Department of Biomedical Engineering, Johns Hopkins University, Baltimore, MD 21205, U.S.A., nitishejhu. edu

Marc H. Schieber is with the Department of Neurobiology and Anatomy, University of Rochester Medical Center, Rochester, NY 14627, mhs@cvs.rochester.edu

${ }^{1}$ Corresponding author: Mayuresh V. Kothare is with the Chemical Process Modeling and Control Center, Department of Chemical Engineering, Lehigh University, Bethlehem, PA 18015, U.S.A. mayuresh.kothare@lehigh.edu that proprioceptive feedback carries kinematic and dynamic information of a natural limb back to the brain through natural afferent pathways. In the case of amputees, this feedback pathway is lost. Therefore BMIs are considered as an open-loop or partially closed-loop system in their current formulations, where the proprioception is either neglected or partially considered using the subject's natural limb [3]. Also in the absence of proprioception, the brain is unable to make error corrections during the voluntary movement of the prosthetic device subject to external loads. Therefore, to improve the versatility of movement in case of amputees, it is necessary to include artificial proprioceptive feedback from the artificial/prosthetic limbs to the brain.

Recently, micro-stimulation techniques have emerged as a promising approach in providing artificial proprioceptive feedback by stimulating appropriate sensory areas of the brain [4]. Even though these techniques are promising for developing future BMIs, the experimental trial and error approach in designing appropriate stimulating sensory input currents may damage a part of the brain or change the natural functionality of the brain. Therefore, a systematic approach that uses optimal feedback control theory is highly desirable towards developing stimulation enhanced next generation BMIs. This approach provides flexibility in designing optimal stimulating sensory input currents and in analyzing the closed-loop neural prosthetic system under various feedback scenarios. It allows modulations in spiking activities of neurons by designing stimulating input currents and simultaneously studying their effect on the entire system in a controlled environment. Moreover, this framework may allow one to obtain an enriched understanding of how our brain works. Motivated by these facts, we develop an optimal feedback control framework based on the receding horizon control architecture [5] for extension/flexion of a finger using activities of a single cortical motor neuron.

Fig. 1 shows the block diagram representation of the receding horizon based optimal feedback control design for the actuation and control of a prosthetic finger in a closedloop framework. Here, "Intent" represents the direction and the goal of the movement. We assume that this intent information is available to the controller prior to design of the closed-loop system. "Receding Horizon Controller" represents the external controller. This controller designs appropriate "stimulating input current" to stimulate "Cortical Motor Neuron". The loop is closed by including "Feedback Currents" available from the system. Feedback information available from the "Torque" and the "Movement" of the "Prosthetic Finger" represents the proprioceptive feedback. The feedback information available from the "Eye" repre- 
sents the visual feedback.

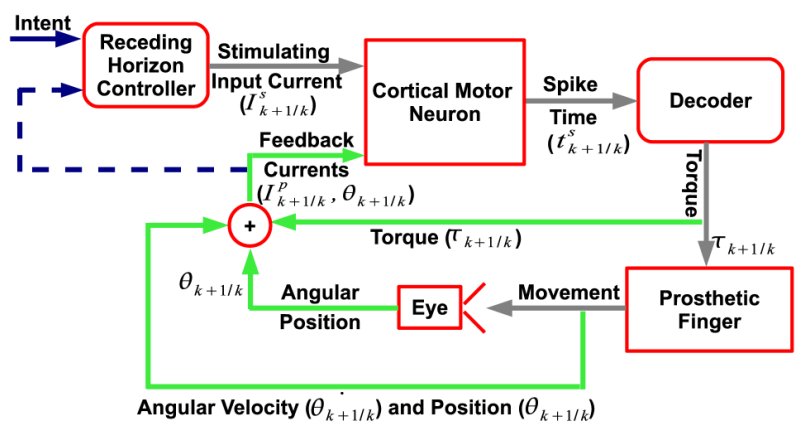

Fig. 1. Receding Horizon based Optimal Neural Prosthetic Finger Control

Although we develop/use computationally efficient and simplified models to elucidate effects of visual and proprioceptive feedbacks in the closed-loop neural prosthetic system, this control framework is general enough to incorporate experimentally validated neurophysiology-based models of the system. The remaining paper is organized as follows. In section II, we develop the control problem and describe our assumptions on the system models. This is followed by feasibility analysis and stability of the receding horizon problem in section III. The formulation of nonconvex nonlinear constrained optimization problem and approach to solve this optimization problem is described in section IV. Then we present a numerical solution and provide results to demonstrate extension of right hand index finger movement in section V. The paper ends with a summary.

\section{Problem Statement}

Receding Horizon Control Problem: We consider an optimal closed-loop neuroprosthetic control problem of the form

$$
\min _{I_{k+1 \mid k}^{s}, l_{k+2 \mid k}^{S}, \cdots, I_{k+N_{c}(k) \mid k}^{s}} \mathbb{J}_{p}(k)
$$

such that

$$
\begin{gathered}
\Delta t_{i \mid k}^{m} \leq \Delta t_{i \mid k}^{s} \leq T_{f} \text { for } k+1 \leq i \leq k+N_{c}(k), \\
\left(\theta_{f \mid k}-\theta_{k+N_{p}(k) \mid k}\right)^{2} \leq \varepsilon .
\end{gathered}
$$

The cost function $\mathbb{J}_{p}(k)$ is defined as $\mathbb{J}_{p}(k)=T_{f}+$ $\sum_{i=k+1}^{k+N_{p}(k)} \Delta t_{i \mid k}^{s}$ at each discrete index $k \geq 0$ and represents a minimum time objective. $i \mid k$ denotes the $i^{t h}$ event at the index $k . N_{p}(k)$ and $N_{c}(k)$ are time varying prediction and control horizon respectively. $N_{p}(k) \geq N_{c}(k), \forall k \geq 0$. $I_{k+1 \mid k}^{s}, \cdots, I_{k+N_{c}(k) \mid k}^{s}$ are stimulating input currents over the control horizon. We set $I_{k+N_{c}(k)+j \mid k}^{S}=I_{k+N_{c}(k) \mid k}^{S}$ for $j=$ $\left\{1,2, \cdots, N_{p}(k)-N_{c}(k)\right\}$. The relationship between these input currents with the cost function $\mathbb{J}_{p}(k)$ is established through the Izhikevich model [6] of a single cortical motor neuron.

Izhikevich Cortical Motor Neuron Model:

$$
\begin{gathered}
\dot{v}(t)=F(v(t))-u(t)+I_{i \mid k}^{s}+I_{i \mid k}^{p}(t), \\
\dot{u}(t)=a(b v(t)-u(t)),
\end{gathered}
$$

$$
\begin{aligned}
& \text { if } \quad v(t) \geq v_{f}, \quad \text { then } \\
& v(t) \leftarrow c \quad \text { and } \quad u(t) \leftarrow u(t)+d .
\end{aligned}
$$

Here, $F(v(t))=0.04 v(t)^{2}+5 v(t)+140 . v(t)$ is the membrane potential (in millivolt $(\mathrm{mV})$ ) at real time $t$ (in millisecond (ms)). $u(t)$ is the membrane recovery variable. The discrete index $i$ belongs to $\left\{k+1, \cdots, k+N_{p}(k)\right\} . I_{i \mid k}^{p}(t)$ is the proprioceptive feedback input current. $v_{f}=30 \mathrm{mV}$ is the cutoff membrane potential. $a, b, c, d$ are dimensionless model parameters. At $\mathrm{t}=0, v(0)=c$ and $u(0)=d$. Whenever the membrane potential $v(t)$ exceeds the cut-off potential $v_{f}$, the occurrence of an action potential is assumed. We define $t_{i \mid k}^{s}$ as the time of the $(i \mid k)^{t h}$ action potential with $t_{1 \mid 0}^{s}=0$. The time duration between the two consecutive action potentials, i.e. the $(i \mid k)^{t h}$ and $(i+1 \mid k)^{t h}$ action potential, is called the $(i \mid k)^{t h}$ inter-spike interval (ISI) and is defined as $\Delta t_{i \mid k}^{s}=t_{i+1 \mid k}^{s}-t_{i \mid k}^{s}$. Thus we establish the relation of $\Delta t_{i \mid k}^{s}$ in the cost function $\mathbb{J}_{p}(k)=T_{f}+\sum_{i=k+1}^{k+N_{p}(k)} \Delta t_{i \mid k}^{s}$ with $I_{i \mid k}^{S}$.

The proprioceptive feedback current $I_{i \mid k}^{p}$ carries information about the rate of contraction of muscle fibers as well as the joint movement. Therefore, we model the proprioceptive feedback current as $I_{i \mid k}^{p}(t)=K_{a} \tau_{i \mid k}(t)+K_{p} \dot{\theta}_{i \mid k}(t) . K_{a}$ and $K_{p}$ are constant gain parameters. $\dot{\theta}_{i \mid k}(t)$ is the angular velocity of the prosthetic finger at time $t \geq t_{i \mid k}^{s} . \tau_{i \mid k}(t)$ is the torque induced by the $(i \mid k)^{t h}$ action potential at the joint of the prosthetic finger. We establish the relation of the torque $\tau_{i \mid k}(t)$ with the timing of action potentials $t_{i \mid k}^{s}$ by developing a dynamical decoder model.

\section{Dynamical Decoder Model:}

$$
\begin{gathered}
\tau_{i \mid k}(t)=\left\{\begin{array}{ll}
\tau_{i-1 \mid k}\left(t_{i \mid k}^{s}\right)+\left(t-t_{i \mid k}^{s}\right) \alpha & t_{i \mid k}^{s} \leq t \leq t\left(\tau_{i \mid k}^{m}\right) \\
-\left(t-t_{i \mid k}^{s}-T_{f}\right) \beta & t\left(\tau_{i \mid k}^{m}\right) \leq t \leq t_{i+1 \mid k}^{s}
\end{array},\right. \\
t\left(\tau_{i \mid k}^{m}\right)=\frac{t_{i \mid k}^{s} \alpha+\left(T_{f}+t_{i \mid k}^{s}\right) \beta-\tau_{i-1 \mid k}\left(t_{i \mid k}^{s}\right)}{\alpha+\beta} .
\end{gathered}
$$

$\tau_{i-1 \mid k}\left(t_{i \mid k}^{s}\right)$ is the measurement of the torque at the time of the $(i \mid k)^{t h}$ action potential. This torque is induced by the $(i-1 \mid k)^{t h}$ action potential at time $t=t_{i-1 \mid k}^{s}$. We assume that $\tau_{0 \mid 0}\left(t_{1 \mid 0}^{s}\right)=0 . t\left(\tau_{i \mid k}^{m}\right)$ is the time at which the torque induced by the $(i \mid k)^{t h}$ action potential reaches its maximum. $\alpha$ and $\beta$ are model parameters. $T_{f}$ assumes a constant value and defines the maximum time in which the torque induced by the $(i \mid k)^{t h}$ action potential dies out. This torque is then used by the prosthetic finger model to extend/flex the finger about the joint.

\section{Prosthetic Finger Model:}

$$
I^{n} \ddot{\theta}_{i \mid k}(t)=-k^{n} \dot{\theta}_{i \mid k}(t)+\tau_{i \mid k}(t) .
$$

Here, $\theta_{i \mid k}(t)$ is the angular position of the finger at time $t \geq t_{i \mid k}^{s} . \dot{\theta}_{i \mid k}(t)$ is the derivative of $\theta_{i \mid k}(t)$ with respect to time $t$ and represents the velocity of the movement. $I^{n}$ is the moment of inertia of the finger about the joint. $k^{n} \dot{\theta}_{i \mid k}(t)$ is the friction term that captures the frictional loss 
during the movement. Unknown model parameters in Eq. (3) and (4) can be estimated using simultaneous experimental recordings of cortical motor neuron ISIs and the movement of a prosthetic finger.

System Constraints: The constraint (1b) defines the minimum and maximum limit on an ISI and ensures that the movement of the prosthetic finger is continuous and smooth. The minimum limit $\Delta t_{i \mid k}^{m}$ is defined as $\max \left\{\Delta t_{m}, \Delta t\left(\tau_{i \mid k}^{m}\right)\right\}$. $\Delta t_{m}$ captures the refractory period of the cortical motor neuron. This means that the cortical motor neuron cannot fire the next action potential within this time period. $\Delta t\left(\tau_{i \mid k}^{m}\right)$ is the time duration, measured from the time of $(i \mid k)^{t h}$ action potential, in which the torque generated by the $(i \mid k)^{\text {th }}$ action potential reaches its maximum. The constraint (1c) defines a target space around the final angular position to be reached during the voluntary movement of the finger. $\varepsilon \geq 0$ is a user defined parameter. $\theta_{k+N_{p}(k) \mid k}$ is the prediction of the angular position of the prosthetic finger in the next $N_{p}(k)$ ISIs designed by the controller at the index $k . \theta_{f \mid k}=\theta_{f}-\theta_{k \mid k}$ is the desired angular movement of the finger at the index $k$ where $\theta_{f}$ is the desired extension / flexion in the finger assuming that the finger is initially at $0^{\circ}$.

Finally, we model the visual input in the form of the difference between the final and current position of the finger. We include this information in the visual model ("Eye" in Fig. 1) which directly measures the current position of the finger. This information is then used by the controller to design optimal stimulating input current. Eqs. (1a, 1b, 1c, 2, 3 and 4) together form a non-linear optimization problem which needs to be solved at each sample time.

\section{FeasibiLIty And Stability}

In this section, we first analyze the feasibility of the control problem stated in section II at $k=0$.

Definition: We say that the control problem stated in section II is feasible at $k=0$ if there exists a sequence of $I_{i \mid 0}^{S}$ for $i=1,2, \cdots, N_{c}(0)$ and $I_{N_{c}(0)+j \mid 0}^{S}=I_{N_{c}(0) \mid 0}^{S}$ for $j=$ $1,2, \cdots, N_{p}(0)-N_{c}(0)$ and thus a corresponding sequence of $\Delta t_{i \mid 0}^{s}$ for $i=1,2, \cdots, N_{p}(0)$ such that constraints (1b) and (1c) are feasible.

Assumption: There exists a sequence $\Delta t_{i \mid 0}^{s}$ for $i=$ $1,2, \cdots, N_{p}(0)$ which satisfies the constraints.

Claim: There exists a sequence $I_{i \mid 0}^{S}$ for $i=1,2, \cdots, N_{c}(0)$ and $I_{N_{c}(0)+j \mid 0}^{s}=I_{N_{c}(0) \mid 0}^{s}$ for $j=1,2, \cdots, N_{p}(0)-N_{c}(0)$ corresponding to $\Delta t_{i \mid 0}^{s}$ for $i=1,2, \cdots, N_{p}(0)$ respectively.

To show the claim, we consider the following cases:

1) $N_{p}(0)=N_{c}(0)=1$ :

a) Eq. (2) has a unique solution $\left(v\left(t, I_{1 \mid 0}^{s}\right), u\left(t, I_{1 \mid 0}^{S}\right)\right)$ for a given $I_{1 \mid 0}^{S}$ and initial conditions [7]. Further, $v\left(t, I_{1 \mid 0}^{S}\right)$ is a continuous function with respect to its arguments.

b) This implies that there is $I_{1 \mid 0}^{s}$ for a given $t=\Delta t_{1 \mid 0}^{s}$ such that the membrane potential $v\left(t, I_{1 \mid 0}^{s}\right)$ satisfies the relation $v\left(\Delta t_{1 \mid 0}^{s}, I_{1 \mid 0}^{s}\right)=v_{f}$.

c) Moreover, first partial derivatives of $v\left(t, I_{1 \mid 0}^{s}\right)$ exist and are continuous with respect to $t$ and $I_{i \mid 0}^{s}$ [8]. d) $F(v(t))$ in Eq. (2) is convex and $v(t)$ is a monotonically increasing function in the neighborhood of $t=\Delta t_{1 \mid 0}^{s}$.

e) By the implicit function theorem [9], the partial derivative of $v\left(t, I_{1 \mid 0}\right)$ with respect to $t$ at $\Delta t_{1 \mid 0}^{s}$ is non-zero for all $I_{1 \mid 0}^{s} \in\left(0, I_{\max }\right)$ where $I_{\max }$ satisfies $v\left(\Delta t_{1 \mid 0}^{s}, I_{\max }\right)=v_{f}$ for $\Delta t_{1 \mid 0}^{s}=\Delta t_{m}$.

f) Thus $\Delta t_{1 \mid 0}^{s}$ is a continuous function of $I_{1 \mid 0}$.

g) Further, by writing the differential of $v\left(\Delta t_{1 \mid 0}^{s}, I_{1 \mid 0}^{s}\right) \quad$ as $\quad \mathrm{d} v\left(\Delta t_{1 \mid 0}^{s}, I_{1 \mid 0}^{s}\right)=0$ implies $\frac{\mathrm{d} \Delta t_{1 \mid 0}^{s}}{\mathrm{~d} I_{1 \mid 0}^{s}}=$ $\left[-\left(\frac{\partial v(t, I)}{\partial I}\right) /\left(\frac{\partial v(t, I)}{\partial t}\right)\right]_{\Delta t_{10}^{s}, I_{1 \mid 0}^{s}}$.

h) The second order partial derivative of $v(t, I)$ with respect to $t$ at $\left\{\Delta t_{1 \mid 0}^{s}, I_{1 \mid 0}^{s}\right\}$ exists.

i) With the assumption that the second order partial derivative of $v(t, I)$ with respect to $I$ at $\left\{\Delta t_{1 \mid 0}^{s}, I_{1 \mid 0}^{s}\right\}$ also exists, the second order derivative of $\Delta t_{1 \mid 0}^{s}$ with respect to $I_{1 \mid 0}^{s}$ exists.

2) $N_{p}(0)=N_{c}(0) \geq 2$ :

a) Given $\Delta t_{1 \mid 0}^{s}$ and $\Delta t_{2 \mid 0}^{s}$, there are corresponding $I_{1 \mid 0}^{S} \quad$ and $\quad I_{2 \mid 0}^{S} \quad$ respectively which satisfy the relation $\quad v\left(\Delta t_{1 \mid 0}^{s}, I_{1 \mid 0}^{s}, v(0), u(0)\right)=v_{f} \quad$ and $v\left(\Delta t_{2 \mid 0}^{s}, I_{2 \mid 0}^{s}, v\left(\Delta t_{1 \mid 0}^{s}\right), u\left(\Delta t_{1 \mid 0}^{s}\right)\right)=v_{f}$.

b) From Eq. (2), $v(0)=v\left(\Delta t_{1 \mid 0}^{s}\right)=c$ and $u(0)=d$. Also, $u\left(\Delta t_{1 \mid 0}^{s}\right)$ is a continuous function of $\Delta t_{1 \mid 0}^{s}$ and $I_{1 \mid 0}^{s}$.

c) Now by the implicit function theorem, $\Delta t_{2 \mid 0}^{s}$ is a continuous function of $I_{1 \mid 0}^{S}$ and $I_{2 \mid 0}^{S}$.

d) Moreover, first and second order partial derivatives of $\Delta t_{2 \mid 0}^{s}$ with respect to $I_{1 \mid 0}^{S}$ and $I_{2 \mid 0}^{S}$ exist and are continuous.

e) Now by extending above arguments to $N_{p}(0)=$ $N_{c}(0)>2, \Delta t_{N_{p}(0) \mid 0}^{s}$ is a continuous function of $\left\{I_{1 \mid 0}^{s}, I_{2 \mid 0}^{S}, \cdots, I_{N_{p}(0) \mid 0}^{s}\right\}$. Moreover, the first and second order partial derivatives of $\Delta t_{N_{p}(0) \mid 0}^{s}$ with respect to $\left\{I_{1 \mid 0}^{s}, I_{2 \mid 0}^{s}, \cdots, I_{N_{p}(0) \mid 0}^{s}\right\}$ exist and are continuous.

Similarly for $N_{p}(0)>N_{c}(0)$, one can show that $\left\{\Delta t_{N_{c}(0)+1 \mid 0}^{s}, \cdots, \Delta t_{N_{p}(0) \mid 0}^{s}\right\}$ are continuous functions of $\left\{I_{1 \mid 0}^{s}, I_{2 \mid 0}^{S}, \cdots, I_{N_{c}(0) \mid 0}^{S}\right\}$ and their partial derivatives with respect to $\left\{I_{1 \mid 0}^{S}, I_{2 \mid 0}^{S}, \cdots, I_{N_{c}(0) \mid 0}^{S}\right\}$ exist and are continuous. Thus the objective function as well as constraints in the optimization problem are well behaved functions of $\left\{I_{1 \mid 0}^{S}, I_{2 \mid 0}^{S}, \cdots, I_{N_{c}(0) \mid 0}^{S}\right\}$.

Now assuming the feasibility at $k=0$ for the control problem, the control problem will remain feasible $\forall k>0$. At $k=0$, we can find a sequence of $I_{i \mid 0}^{s}$ for $i=1,2, \cdots, N_{c}(0)$ and $I_{N_{c}(0)+j \mid 0}^{s}=I_{N_{c}(0) \mid 0}^{s}$ for $j=1,2, \cdots, N_{p}(0)-N_{c}(0)$ such that the control problem is feasible. At $k=1$, we can choose a set of admissible control inputs [5] $I_{i \mid 0}^{S}$ for $i=2,3, \cdots, N_{c}(0)$ and $I_{N_{c}(0)+j \mid 0}^{S}=I_{N_{c}(0) \mid 0}^{s}$ for $j=1,2, \cdots, N_{p}(0)-N_{c}(0)$, assuming $N_{c}(0)$ is fixed. This set can steer the $\theta_{N_{p}(0) \mid 1}$ to the $\varepsilon$ region of $\theta_{f \mid 1}$. If we continue this way, we find that the control problem is feasible $\forall k>0$ if it is feasible at $k=0$.

Stability: The stability of the control problem can be guaranteed by the terminal set constraint and well behaved dynamics of the control problem. Now we can use Theorem (7.5) from [5] to guarantee that for $N_{p}(k)>1$, there exists 
control horizon pair $\left(\Pi(k), N_{p}(k)\right)$ such that

$$
\mathbb{J}_{p}\left(k, \Pi(k), N_{p}(k)\right) \leq \mathbb{J}_{p}\left(k, \Pi(k)^{\prime}, N_{p}(k)-1\right)
$$

and there exists $\eta(k) \in(0, \infty)$ satisfying

$$
\mathbb{J}_{p}\left(k+1, \Pi(k+1), N_{p}(k+1)\right) \leq \mathbb{J}_{p}\left(k, \Pi(k), N_{p}(k)\right)-\eta(k),
$$

where $\eta(k)=\Delta t_{k+1 \mid k}^{s}, \Pi(k)=\left\{I_{k+1 \mid k}^{s}, I_{k+2 \mid k}^{s}, \cdots, I_{k+N_{p}(k) \mid k}^{s}\right\}$, $\Pi(k)^{\prime}=\left\{I_{k+2 \mid k}^{s}, I_{k+2 \mid k}^{s}, \cdots, I_{k+N_{p}(k) \mid k}^{S}\right\}$ and $I_{k+j \mid k}^{s}=I_{k+N_{c}(k) \mid k}^{s}$ for $j=1,2, \cdots, N_{p}(k)-N_{c}(k)$.

\section{NON-LINEAR Optimization Problem}

In this section, we write the appropriate first order KarushKuhn-Tucker (KKT) conditions for the non-linear optimization problem represented by Eqs. (1a, 1b and 1c). It should be noted here that the optimization algorithm is the same for all $k$. Therefore, we focus on the optimization problem at $k=0$ and write the KKT conditions at $k=0$. Basic requirements for the KKT optimality conditions are that the Lagrangian function should be twice continuously differentiable with respect to optimization variables. At $k=0$, it is clear from the section III that the objective function, $\mathbb{J}_{p}(0)=T_{f}+\sum_{i=1}^{N_{p}(0)} \Delta t_{i \mid 0}^{s}$, and the constraint, defined by Eq. (1b), are twice continuously differentiable with respect to $\left\{I_{1 \mid 0}^{s}, I_{2 \mid 0}^{s}, \cdots, I_{N_{c}(0) \mid 0}^{S}\right\}$ for $i=1,2, \cdots, N_{p}(0)$. To show continuous differentiability of the constraint (1c) with respect to $\left\{I_{1 \mid 0}^{s}, I_{2 \mid 0}^{S}, \cdots, I_{N_{c}(0) \mid 0}^{S}\right\}$, we provide the following argument. From the dependency of $\tau_{i \mid 0}$ on $\Delta t_{i \mid 0}^{s}$ in Eq. (3), it is clear that $\theta_{N_{p}(0) \mid 0}$ is a smooth function of $\Delta t_{i \mid 0}^{s}$, where $i=1, \cdots, N_{p}(0)$ and thus at least twice continuously differentiable with respect to $\left\{I_{1 \mid 0}^{s}, \cdots, I_{N_{c}(0) \mid 0}^{S}\right\}$.

To solve the optimization problem, we implemented the infeasible interior point algorithm as proposed in [10]. For this, we wrote $\mathbf{h}\left(\mathbf{I}_{\mathbf{0}}^{\mathbf{s}}\right)=\left[\Delta t_{1 \mid 0}^{s}-\Delta t_{1 \mid 0}^{m}, \cdots, \Delta t_{N_{c}(0) \mid 0}^{s}-\Delta t_{N_{c}(0) \mid 0}^{m}, T_{f}-\right.$ $\left.\Delta t_{1 \mid 0}^{s}, \cdots, T_{f}-\Delta t_{N_{c}(0) \mid 0}^{s}, \mathcal{E}-\left(\theta_{f \mid 0}-\theta_{N_{p}(0) \mid 0}\right)^{2}\right]^{T}$, where $(\cdot)^{T}$ denotes the transpose. We reformulated the optimization problem as follows:

$$
\min _{\mathbf{I}_{\mathbf{0}}, \mathbf{w}}\left(\mathbb{J}_{p}(0)-\mu \sum_{i=1}^{2 N_{c}(0)+1} \log w_{i}\right)
$$

subject to

$$
\mathbf{h}\left(\mathbf{I}_{\mathbf{0}}^{\mathbf{s}}\right)-\mathbf{w}=\mathbf{0},
$$

where $w_{i}, i=1,2, \cdots, 2 N_{c}(0)+1$ are positive slack variables, $\mu$ is the barrier parameter, $\mathbf{w}=\left[w_{1}, \cdots, w_{2 N_{c}(0)+1}\right]^{T}$ and $\mathbf{I}_{\mathbf{0}}^{\mathbf{S}}=$ $\left[I_{1 \mid 0}^{S}, \cdots, I_{N_{c}(0) \mid 0}^{S}\right]^{T}$. The first order KKT conditions for the minimum are given by following equations:

$$
\left[\begin{array}{c}
\nabla \mathbb{J}_{p}(0)-\nabla \mathbf{h}\left(\mathbf{I}_{\mathbf{0}}^{\mathbf{s}}\right)^{T} \lambda \\
-\mu \mathbf{W}^{-1} \mathbf{e}+\lambda \\
\mathbf{w}-\mathbf{h}\left(\mathbf{I}_{\mathbf{0}}^{\mathbf{s}}\right)
\end{array}\right]=0
$$

where $\mathbf{W}$ is a diagonal matrix with elements of $\mathbf{w}, \lambda$ is a vector of non-negative Lagrange multipliers, $\mathbf{e}$ is the unit vector of length $2 N_{c}(0)+1$. To solve the first order KKT condition Eq. (8) numerically, we applied the standard
Newton method. In the symmetric form, the Newton update for Eq. (8) becomes

$$
\begin{array}{r}
{\left[\begin{array}{cc}
-\mathbf{H}(0) & \nabla \mathbf{h}\left(\mathbf{I}_{\mathbf{0}}^{\mathbf{s}}\right)^{T} \\
\nabla \mathbf{h}\left(\mathbf{I}_{\mathbf{0}}^{\mathbf{s}}\right) & \mathbf{W} \Lambda^{-1}
\end{array}\right]\left[\begin{array}{c}
\Delta \mathbf{I}_{\mathbf{0}}^{\mathbf{s}} \\
\Delta \lambda
\end{array}\right]=\left[\begin{array}{c}
\nabla \mathbb{J}_{p}(0)-\nabla \mathbf{h}\left(\mathbf{I}_{\mathbf{0}}^{\mathbf{s}}\right)^{T} \lambda \\
\mathbf{c}\left(\mathbf{I}_{\mathbf{0}}^{\mathbf{s}}\right)+\mathbf{W} \Lambda^{-1}\left(\mu \mathbf{W}^{-1} \mathbf{e}-\lambda\right)
\end{array}\right],} \\
\Delta \mathbf{w}=\mathbf{W} \Lambda^{-1}\left(\mu \mathbf{W}^{-1} \mathbf{e}-\lambda-\Delta \lambda\right),
\end{array}
$$

where $\mathbf{H}(0)=\nabla^{2} \mathbb{J}_{p}(0)-\sum_{i=1}^{2 N_{c}(0)+1} \lambda_{i} \mathbf{h}_{i}\left(\mathbf{I}_{\mathbf{0}}^{\mathbf{s}}\right), \quad \mathbf{c}\left(\mathbf{I}_{\mathbf{0}}^{\mathbf{s}}\right)=\mathbf{w}-$ $\mathbf{h}\left(\mathbf{I}_{\mathbf{0}}^{\mathbf{s}}\right) . \Lambda$ is the diagonal matrix with elements of $\lambda . \Delta \mathbf{I}_{\mathbf{0}}^{\mathbf{s}}, \Delta \lambda$ and $\Delta \mathbf{w}$ are the Newton steps in $\mathbf{I}_{\mathbf{0}}^{\mathbf{s}}, \lambda$ and $\mathbf{w}$ respectively. To find the descent directions for the Newton steps, we implemented the Markov filter method [11]. Detailed description of the algorithm is given in [10], [11].

\section{NuMERICAL RESUlts}

In this section, we study the control problem for four systems namely closed-loop, partial closed-loop-V, partial closedloop-P and open-loop. Table I shows the distinction among these systems.

TABLE I

SySTEM DESIGN

\begin{tabular}{|l|c|r|}
\hline System & Visual feedback & Proprioceptive feedback \\
\hline \hline Closed-loop & Yes & Yes \\
\hline Partial closed-loop-V & Yes & No \\
\hline Partial closed-loop-P & No & Yes \\
\hline Open-loop & No & No \\
\hline
\end{tabular}

The Izhikevich cortical neuron model parameters $a, b, c, d$ were set to $0.0404,0.2497,-64.4679,21.2777$ respectively. These parameters were computed using the experimental data recorded from a cortical motor neuron during the right hand index finger movement in a primate study [12]. $\Delta t_{m}$ was set to $2 \mathrm{~ms}$. The model parameters $\alpha, \beta, T_{f}$ in Eq. (3) were set to $0.5,0.01,50$ respectively. These choices of parameter allowed us to extend the index finger by $20^{\circ}$ in the time interval of $1-2 \mathrm{~s}$. For systems with visual feedback, initial value of $\varepsilon$ was set to 0.2 . $\varepsilon$ was monotonically decreased during the receding horizon problem and finally fixed to 0.01. For systems without visual feedback, $\varepsilon$ was set to 0.01 . Thus the final target location of $\theta$ was $19.9^{\circ}-20.1^{\circ}$. The control horizon $N_{c}(0)$ was set to 4 . Initial guesses for $\left\{I_{1 \mid 0}, \cdots, I_{N_{c}(0) \mid 0}\right\}$ were set to 25 . The initial prediction horizon $N_{p}(0)$ was computed such that the constraint Eq. (1c) and thus the receding horizon control problem becomes feasible at initial guesses. It should be noted here that the prediction horizon $N_{p}(k)$ was not decreased monotonically during the receding horizon computation. Eqs. (2, 3 and 4) were computed numerically using the Euler scheme with the fixed step size of $10^{-3}$.

We solved the receding horizon control problem described in the section II at each $k \geq 0$. For this, we implemented the optimization algorithm, shown in the section IV, in the MATLAB programming environment. We set the step length parameter and the settable scale factor in the optimization algorithm to their default values, which are given in [10]. Initial values of $\mathbf{w}$ were set to $\mathbf{h}(\mathbf{I})$. Initial values of the Lagrange multipliers were set to $\lambda_{i}=1 / \mathbf{h}_{i}$ for $i=1, \cdots, 2 N_{c}$ and $\lambda_{2 N c+1}=1$. We observed that this particular choice works for almost every problem we solved in our context. With this, 
we analyzed effects of visual and proprioceptive feedback in designing the closed-loop neural prosthetic system.

It has been shown in BMIs studies that visual as well as proprioceptive feedback play important role in guiding voluntary movement tasks such as reaching or grasping [13], [14]. In [14], it has been shown that the difficulty in using a prosthetic limb for grasping a virtual object increases as one or both of these feedback signals are switched off. Particularly, it may even be impossible to attain the task goal in the absence of visual feedback. Here, we studied the importance of visual feedback from a different perspective. We assumed that it is possible to reach the desired extension of the finger in the open loop, partial closed-loop- $\mathrm{V}$ and partial closed-loop-P system under nominal conditions. We set proprioceptive feedback gains $K_{a}$ and $K_{p}$ to 0 for the open-loop and partial closed-loop-V system. For the partial closed-loop-P system, we set $K_{a}=1$ and $K_{p}=100$. With this, we studied the effect of visual feedback in the presence of synaptic noise. We first solved the control problem for the open-loop system and the partial closed-loop-P system and computed optimal stimulating input currents for both systems. This provided us the number of ISIs required to reach the desired angular position in both systems. It is a well known fact that synaptic currents are fundamentally noisy. Therefore we introduced a synaptic noise model (the Gaussian noise with mean 0 and variance $\sigma^{2}$ ) into the cortical motor neuron model Eq. (2). We implemented the computed optimal stimulating input currents on this stochastic model with $\sigma=20$ and obtained same number of ISIs as in the absence of noise. Using these ISIs, we computed the final angular position reached by the finger in the presence of noise. The top plot in Fig. 2 represents the angular trajectory with respect to time for the open-loop system in the absence and presence of synaptic noise as well the partial closedloop-P system in the presence of synaptic noise.
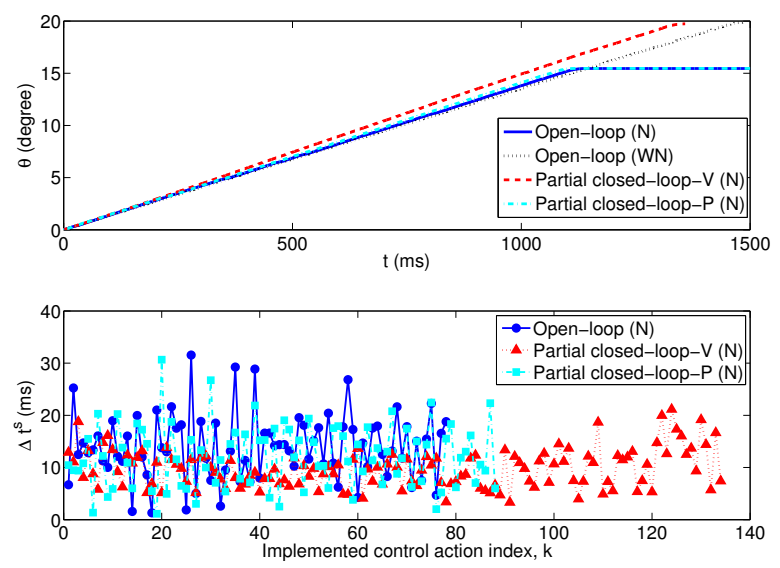

Fig. 2. Importance of visual feedback in designing neural prosthetic system: Here, the top plot shows the angular position trajectory during the extension of the finger. The $y$-axis represents the angular position $(\theta$ measured in degrees) and the $\mathrm{x}$-axis represents the time ( $t$ in milliseconds). "N" indicates the presence of synaptic noise during the design, "WN" indicates absence of any synaptic noise during the design. The bottom plot represents modulations in inter-spike intervals (ISIs) of the cortical motor neuron in the absence and presence of visual feedback information.
Here the angular trajectory of the open-loop system, "Openloop (N)", and partial closed-loop system, "partial closedloop-P $(\mathrm{N})$ ", in the presence of synaptic noise clearly shows that the desired angular position is not reached in both cases. Therefore, to reach the desired angular position in the presence of synaptic noise, it is necessary to modify the number of ISIs. This modification is only possible using the available information of the prosthetic finger angular position through the visual feedback. To show this, we solved the control problem for the partial closed-loop system. We computed optimal stimulating input currents over the control horizon at each discrete step for the nominal system and implemented the first computed stimulating current on the stochastic neuron model. With the computed ISI using this model, we computed the present angular position and used this information as a visual feedback in designing the next stimulating input current. The angular trajectory for this system is shown in the top plot of Fig. 2 as "Partial closedloop (N)".

The bottom plot in Fig. 2 shows the modulation in cortical activities of the neuron in the presence of synaptic noise for the open-loop, the partial closed-loop- $\mathrm{V}$ and the partial closed-loop-P system. It indicates that the visual feedback helps the brain in rejecting the effect of synaptic noise during the extension of the finger by modifying cortical activities of the neuron in the partial closed-loop system. Next, we study the role of proprioception in a closed-loop neural prosthetic system.

Experimental evidence indicates that there is a direct relation between the muscle force and activities of cortical motor neurons [13], [15]. Particularly, in [13], it has been shown that proprioception affects firing rates of cortical motor neurons significantly and enhances performance of BMIs in on-line operation. Therefore, here, we studied firing rate of the cortical motor neuron in the closed-loop and the partial closed-loop- $\mathrm{V}$ designs in the absence of synaptic noise and investigated the effect of proprioception on the firing rate of the cortical motor neuron. For this, we assumed that it is possible to reach the desired extension of the finger in the absence and presence of proprioceptive feedback. We set proprioceptive feedback gains $K_{a}$ and $K_{p}$ to 1 and 100 respectively. Model parameters as well as number of control horizon were same for both systems as defined at the beginning of this section. Fig. 3 shows modulation in ISIs of the cortical motor neuron over the prediction horizon for the closed-loop system and the partial closed-loop-V system in the receding horizon based optimal control framework.

Fig. 3 clearly shows that ISIs of the cortical motor neuron decreased after including the proprioceptive feedback in the design. It is also shown in Fig. 3 that these decrements in ISIs are not significant to conclude necessity of proprioception in the operation of neural prosthetic system. We suspect that a possible reason for this small decrement may be the small gain value of $K_{a}$ used in the proprioceptive feedback. Therefore, next, we studied how activities of the cortical motor neuron change as we change gains in the proprioceptive feedback. 


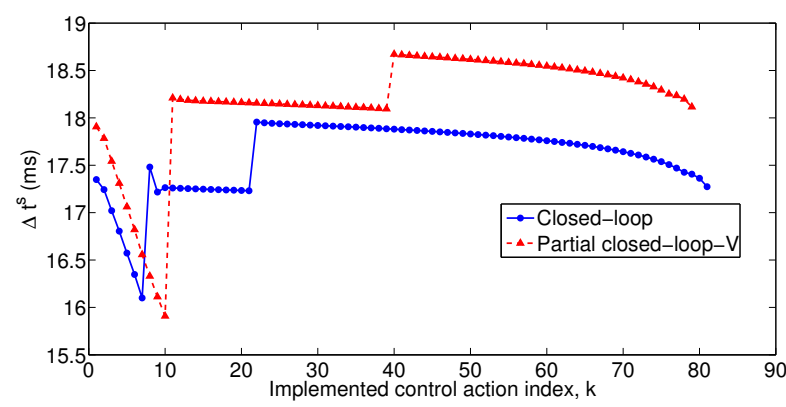

Fig. 3. Modulations in inter-spike intervals (ISIs) of the cortical motor neuron in presence and absence of proprioception for a task of extending a finger by $20^{\circ}$ about a joint: Here the y-axis represents ISIs variations $\left(\Delta t^{s}\right.$ in milliseconds) and the $\mathrm{x}$-axis represents the control action index $(k)$.

It was shown in a classical experiment by Evarts [15] that the change in activities of cortical motor neurons signal the amplitude of muscle force required to produce movement. In particular, increase in external load opposing the direction of movement increases firing rate of cortical motor neurons. Therefore, we investigated the effect of torque sensed by proprioception on the activities of the cortical motor neuron. For this, we fixed the gain $K_{p}$ to a value of 100 and varied the gain $K_{a}$. It should be noted that the gain $K_{a}$ carries torque information in the proprioceptive feedback current model and resembles the perception of extra force required by the muscle. To reduce the computational effort, we set the parameter $\alpha$ in Eq. (3) to 2 so that we reached the desired angular position of $10^{\circ}$ in less than 25 prediction steps. With this setup, we studied variations in ISIs as a function of the gain $K_{a}$. Fig. 4 represents how ISI vary over prediction horizon for three different values of $K_{a}$ in the closed-loop system.

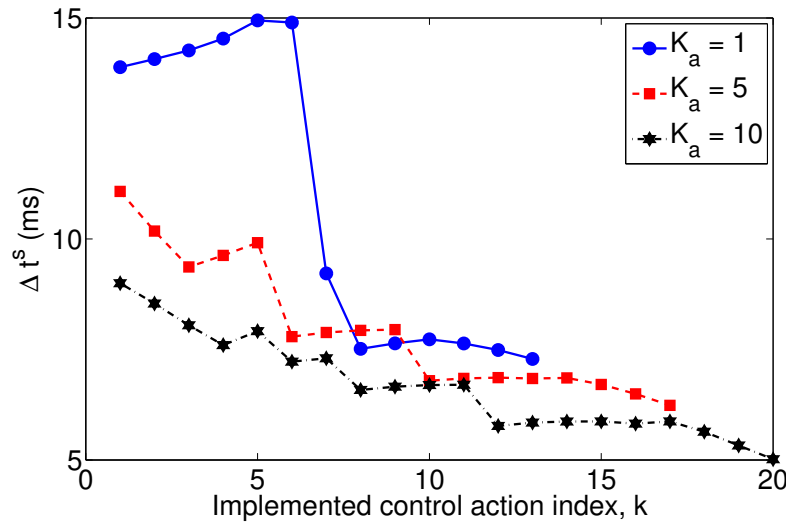

Fig. 4. Modulations in inter-spike intervals (ISIs) of the cortical motor neuron in presence of three different proprioceptive feedbacks for a task of extending a finger by $10^{\circ}$ about a joint: Here the closed-loop system includes proprioception as well visual feedbacks, increasing values of the gain $K_{a}$ resemble more perception of the required torque for accomplishing the task, the y-axis represents ISIs variations ( $\Delta t^{s}$ in milliseconds) and the $\mathrm{x}$-axis represents the control action index $(k)$.

Fig. 4 shows that ISIs of the cortical motor neuron changed significantly when we increased the torque perceived by the proprioceptive feedback current.

\section{SUMMARY}

In summary, we have developed a generalized optimal control framework to study closed-loop system behavior in neuroprosthetics applications. To the best of our knowledge, this is the first systematic attempt to formalize closed-loop neuroprosthesis analysis in an optimal predictive control framework. Our results clearly indicate significant advantages of using an optimal control framework in studying complex biological systems such as neural prosthetic systems which may be difficult in an experimental framework. Our findings suggest that

1) visual feedback is important in rejecting internal noises, naturally occurs in neuronal network, while reaching the desired goal of the task;

2) proprioceptive feedback increases firing rate of the single cortical motor neuron.

\section{ACKNOWLEDGEMENT}

Financial support from the US National Science Foundation, Cyber Enabled Discovery and Innovation (CDI) program, is gratefully acknowledged.

\section{REFERENCES}

[1] M. A. L. Nicolelis and M. A. Lebedev, "Principles of neural ensemble physiology underlying the operation of brain-machine interfaces," Nature Reviews, vol. 10, pp. 530-540, 2009.

[2] S. Koyoma, S. M. Chase, A S. Whitford, M. Velliste, A. B. Schwartz, and R. E. Kass, "Comparison of brain computer interface decoding algorithms in open-loop and closed-loop control," Journal of Computational Neuroscience, vol. 29, no. 1-2, pp. 73-87, August 2010.

[3] J. P. Cunnigham, P. Nuyujukian, V. Gilja, C. A. Chestek, S. I. Ryu, and K. V. Shenoy, "A closed-loop human simulator for investigating the role of feedback-control in Brain Machine Interfaces," Journal of Neurophysiology, vol. 105, pp. 1932-1949, 2011.

[4] N. A. Fitzsimmons, W. Drake, T. L. Hanson, M. A. Lebedev, and M. A. L. Nicolelis, "Primate reaching cued by multichannel spatiotemporal cortical microstimulation," The Journal of Neuroscience, vol. 27, no. 21, pp. 5593-5602, 2007.

[5] W. H. Kwon and S. Han, Receding Horizon Control, Springer, 2005.

[6] E. M. Izhikevich, "Which model to use for cortical spiking neurons?," IEEE Transactions on Neural Networks, vol. 15, pp 1063-1070, 2004.

[7] J. Touboul, "On the simulation of nonlinear bidimensional spiking neuron model," Preprints, 2010.

[8] H. K. Khalil, Nonlinear Systems, Prentice Hall, 2002.

[9] T. M. Apostol, Mathematical Analysis, Addison-Wesley Mathematics Series, 1957

[10] R. J. Vanderbei and D. F Shanno, "An interior-point algorithm for nonconvex nonlinear programming," Computational Optimization and Applications, vol. 13, pp 231-252, 1999.

[11] H. Y. Benson, D. F Shanno and R. J. Vanderbei, "Interior-point methods for nonconvex nonlinear programming: Filter methods and merit functions," Computational Optimization and Applications, vol. 23, no. 2, pp 257-272, 2002.

[12] G. Kumar, V. Aggarwal, N. V. Thakor, M. H. Schieber and M. V. Kothare, "Optimal parameter estimation of the Izhikevich single neuron model using experimental inter-spike interval (ISI) data," In Proceedings of the 2010 American Control Conference, Baltimore, MD, pp. 3586-3591, 2010.

[13] A. J. Suminski, D. C. Tkach, A. H. Fagg and N. G. Hatsopoulos "Incorporating feedback from multiple sensory modalities enhances brain-machine interface control," The Journal of Neuroscience, vol. 30, no. 50, pp. 16777-16787, December 2010.

[14] A. Blank, A. M. Okamura and K. J. Kuchenbecker "Identifying the role of proprioception in upper-limb prosthesis control: studies on targeted motion," ACM Transactions on Applied Perception, vol. 7 , no. 3, pp. 1-23, June 2010.

[15] E. R. Kandel, J. H. Schwartz and T. M. Jessell, Principles of Neural Science, Forth Edition, McGraw-Hill, 2000. 